\title{
Design and Implementation of the Water Environment Monitoring and Management System Based on WebGIS for the Four-Lake Basin of Hubei Province
}

\author{
Kai Luo ${ }^{1,4}$, Ting Zhang ${ }^{1,2,3^{*}}$, and Yanwen $\mathrm{Liu}^{1}$ \\ ${ }^{1}$ Hubei University of Science and Technology, School of Resources Environmental Science and Engineering, 437100 Xianning, China; \\ ${ }^{2}$ Anhui Jianzhu University, School of Environment and Energy Engineering, 230601 Hefei, China; \\ ${ }^{3}$ Key Laboratory of Huizhou Architecture in Anhui Province, 230601 Hefei, China; \\ ${ }^{4}$ China University of Geosciences, School of Geography and Information Engineering, 430074 Wuhan, China
}

\begin{abstract}
The river network in the Four-lake Basin of Hubei Province is staggered with a complicated water system and is seriously disturbed by human activities. Therefore, the real-time monitoring and management of its water environment is of great significance to the sustainable development of the basin. As the application of WebGIS becoming increasingly extensive, applying WebGIS to the monitoring and management of basin water environment can satisfy the demands of various users, such as governmental departments, general users, and researchers in the context of increasingly-prominent water environment problems in the basin. The WebGIS technology has utilized to realize the functions of displaying, spatially querying and analyzing water environment basic information such as hydrology, water quality, meteorology and natural reserves in the Four-lake Basin. Users can easily access and obtain relevant real-time dynamic information through the browser for spatial analysis, such as the assessment of water eutrophication and the diffusion analysis of wastewater pollutants.
\end{abstract}

\section{Introduction}

The health of water environment in the basin is an important foundation for developing regional economics and production activities ${ }^{[1-2]}$. A great number of WebGIS platforms concerning water ecological environment, such as water resources assessment ${ }^{[3]}$, soil and water conservation ${ }^{[4]}$, flood prevention and dispatching system ${ }^{[5]}$, have emerged at home and abroad, aiming at promoting the sharing, comprehensive application and integrated analysis of water environment data. Most of these systems can provide the functions of querying and analyzing, such as querying and downloading water environment data ${ }^{[6-7]}$, while few of them can have more spatial analysis functions, such as buffer analysis, geostatistical analysis, and inverse distance weighting analysis ${ }^{[8-9]}$. This study took the case study of the water environment monitoring system of the Four-lake Basin as an example, and utilized the WebGIS technology to realize the functions of displaying, spatially querying, and analyzing the basic information of the water environment, such as hydrology, water quality, meteorology, and natural reserves in the Fourlake Basin. Users can easily access and obtain relevant real-time dynamic information through the browser for spatial analysis such as the assessment of water eutrophication. The study can provide a strong support for the scientific decision-making of the river basin management department, general users, and environment assessment analysis.

\section{Technical methods and system architecture}

\subsection{Technical methods and routes}

The integrity, scalability and modularity of the water environment system platform have been considered in the technical route established in the system. The major technical platforms and methods adopted are shown as follows:

(1) The GIS platform was ArcGIS for Desktop, through which the visual data information or map feature information was obtained. Then, based on this information, map spatial analyses, including buffer analysis, geostatistical analysis, topological analysis, and inverse distance weighting analysis, were performed.

(2) For the spatial database, a large relational spatial database, SQL server2012+SDE was adopted for storing spatial data and attribute data.

(3) A highly responsive, convenient, and userfriendly $\mathrm{B} / \mathrm{S}$ (browser/client) system structure was adopted in the system. Data from various government and non-government departments were integrated into system applications. The map server and the map engine

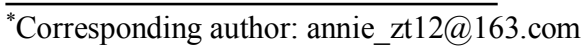


used were ArcGIS for Server and ArcGIS API for JavaScript.

(4) An object-oriented development approach was adopted for procedure design to ensure that the system meets the description of user's needs to the greatest extent, allowing the intelligentization and design of related functional modules to be more universal.

(5) Concerning the technical use of the back-end platform, the Java language and SSM framework were adopted. Object-oriented analysis and design methods were utilized, while the commonly-used three-tier structure system consisting of the presentation layer, the business layer, and the data layer were adopted.

\subsection{System architecture}

The system architecture was composed of client services, map server services, and data operation services (Fig. 1); their interactions were realized through Ajax requests. Moreover, the front-end business was separated from the back-end logic. Meanwhile, interface encapsulation was adopted to call the front end in the service of querying general attributes and the map service.

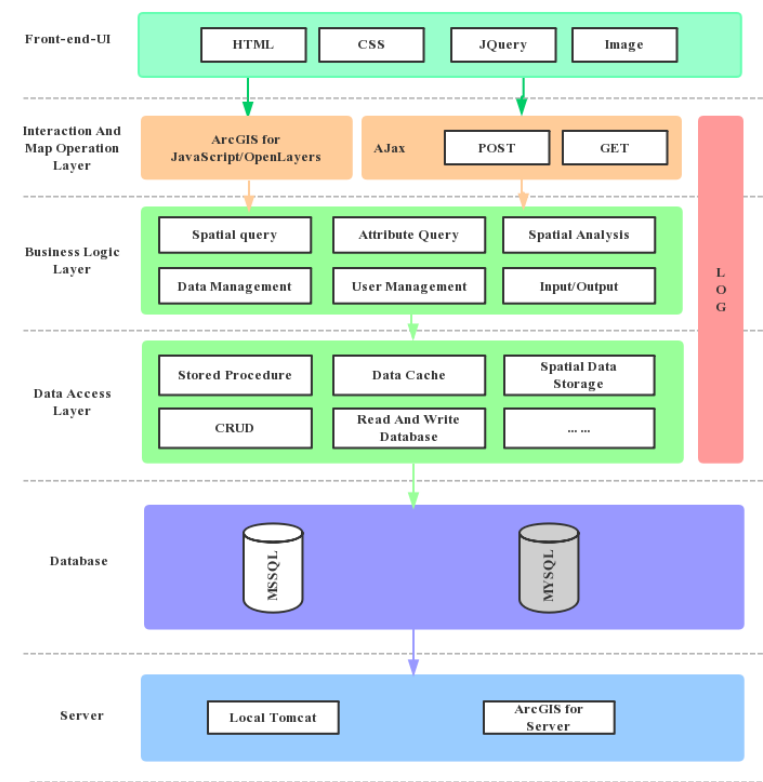

Fig. 1. System architecture diagram

\section{Overall system design}

The overall design of the system consists of preliminary design, functional module design, and interface design. The composition of functional modules of the water environment monitoring system of the Four-lake Basin, the overall framework of the system, the source of data, and the relationship between various parts was described.

\subsection{System function modules}

The main functional modules of the Water Environment Monitoring System for the Four-lake Basin include basic map functions, spatial query functions, attribute query, spatial analysis, input/ output, and data management, etc. as shown in Fig. 2.

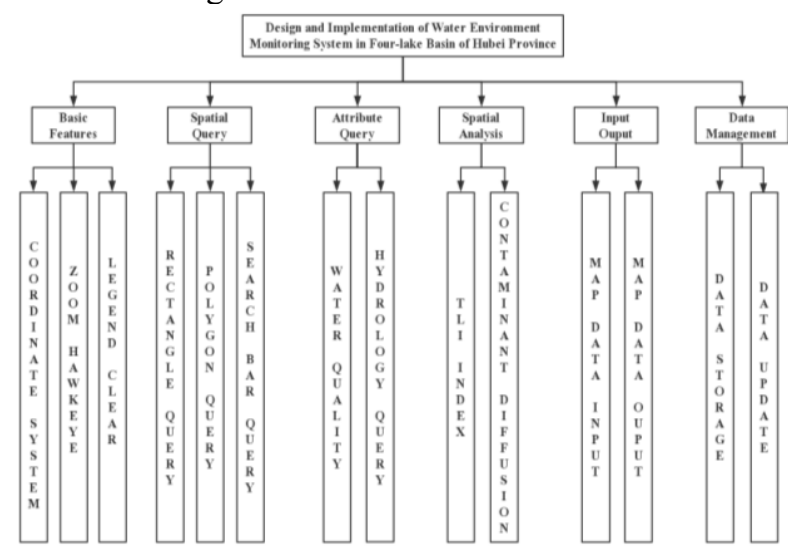

Fig. 2. System function module diagram

\subsection{GIS interface design}

The interface design of GIS mainly involves the geospatial operation interface and the attribute operation interface (Table 1). The function of the geospatial operation interface to provide querying buffer and querying geometry on ArcGIS for Server, whereas that of the attribute operation interface consists of querying data indexes, such as water quality and meteorology.

Table 1. System GIS interface

\begin{tabular}{|c|c|}
\hline Interface name & Function and description \\
\hline ILoginDao & DAO Database operation \\
\hline$/$ GeoLakes/h/query & Water quality information query \\
\hline /GeoLakes/t/getData & $\begin{array}{c}\text { Store hydrological information in } \\
\text { the database }\end{array}$ \\
\hline /GeoLakes/n/query & Nature reserve information query \\
\hline
\end{tabular}

\subsection{Data source}

The data required by the system primarily originated from the Internet, including Jingzhou Hydrological Information Sub-Center, Blue Map Data Center, and National Meteorological Information Center. The data were acquired using Python and the Scarpy crawler framework. Hydrological data between Jan. 1, 2015 and Feb. 24, 2018 have been extracted from the Jingzhou Hydrological Information Sub-Center through crawling. While corporate wastewater discharge data, including indicators such as biochemical oxygen demands, chemical oxygen demands, total nitrogen, total phosphorus, and $\mathrm{pH}$ values, have been extracted the same way from the Blue Map Data Center.

\section{System design specifications}

\subsection{Database design}

The design of the system database involved three phases, including database concept design, database logic design, and database physical design with the Microsoft SQL Server 2012 relational database. These data were then 
stored by means of separating the business data table from the spatial data table. The structure of the data table of the hydrological information table and user login information table in the system is presented in Table 2. and Table 3.

Table 2. Structural information of hydrological information table

\begin{tabular}{|c|c|}
\hline Filed & Field type and length \\
\hline id & $\operatorname{Varchar}(64)$ \\
\hline siteName & Varchar(30) \\
\hline siteLevel & Varchar(10) \\
\hline siteFlow & Varchar(10) \\
\hline Time & Varchar(64) \\
\hline siteDate & Varchar(64) \\
\hline
\end{tabular}

Table 3. Structural information of user login information table

\begin{tabular}{|c|c|}
\hline Filed & Field type and length \\
\hline id & Varchar(64) \\
\hline username & Varchar(64) \\
\hline password & Varchar(30) \\
\hline
\end{tabular}

\subsection{System function design}

\subsubsection{Basic map functions}

The basic map functions of the system include:

(1) Map browsing, translating, zooming, Hawk-eye, scale, latitude/ longitude, and legend, etc.;

(2) Swipe contrast, multi-screen contrast, and element identification;

(3) Search, query, and layer selection, etc.;

(4) Attribute query of the basic attribute table.

\subsubsection{System spatial query}

Spatial query analysis was performed on the main interface of the map. Different spatial query functions were designed as per layer elements. These spatial query functions include displaying the information of the attribute table of the selected elements after the polygon query, displaying the queries and notifications of hydrological information, water quality indicators, and main wastewater discharges, as well as displaying different grade information corresponding to different values according to the national standards. Searched elements were located after the rectangular query and spatial search. Its basic information and attribute table were displayed with popup notifications (Fig. 3).

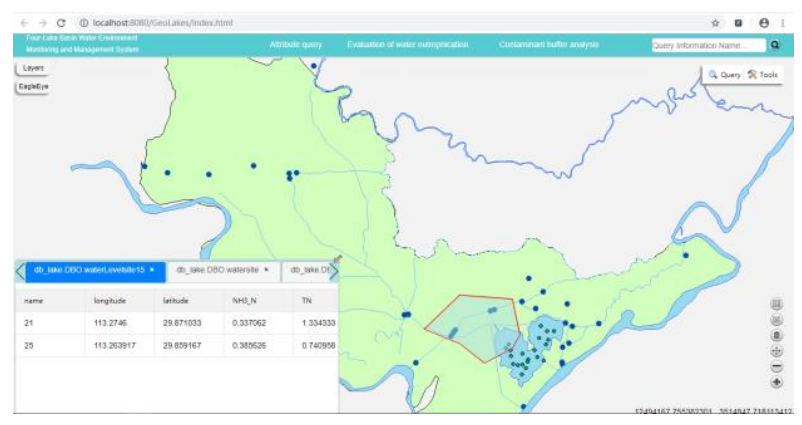

Fig. 3. Polygon query schematic

\subsubsection{Map spatial analyzing function}

(1) Evaluation of water eutrophication

Eutrophication indexes were utilized to conduct spatial query. Water quality index data related to the eutrophication index were obtained through the coordinate information that was gained from the selected water quality points, before it was transferred to the backstage using Ajax. In this way, the eutrophication index could be calculated with the model algorithm of the eutrophication index calculation at the backstage. After that, the indexes were returned to the front end for displaying. The grading of the eutrophication index was determined in accordance with the model standard to obtain the eutrophication grades. By referring to the comprehensive eutrophication status index proposed by Wang et al. ${ }^{[10]}$, the assessment of eutrophication was made based on the concentrations of the following five parameters: chlorophyll a, total phosphorus, total nitrogen, transparency, and permanganate index. The degree of eutrophication of the water body could be assessed in line with the eutrophication index and the eutrophication grade obtained (Fig. 4).

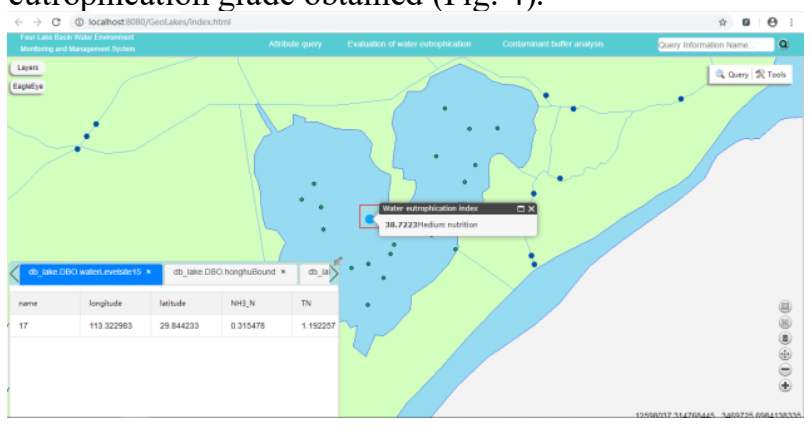

Fig. 4. Water eutrophication evaluation

(2) Wastewater pollution diffusion model

A wastewater discharge diffusion model was established based on the wastewater sources. Meanwhile, buffer analysis and inverse distance weighting interpolation (IDW) were also adopted to predict the concentration of wastewater pollutants at any river points. The algorithm works as follows: the geometric processing service in ArcGIS for Server is firstly utilized to establish a 10-kilometer buffer for any predicted point in the river; next, the geometric service is used for calculating and querying the distance from the wastewater discharge point within the buffer to the predicted point; at last, the concentration of diffused 
pollutants at the predicted point is calculated using the inverse distance weighting interpolation.

\section{Conclusion}

The environment monitoring system of the Four-lake Basin based on the WebGIS service technology was implemented in this study, and elaborations of the technical method, the framework structure, the function module, the overall design, as well as the detailed design of the system were also given. Moreover, the monitoring system has been proven able to provide real-time query of hydrological sites and meteorological sites, query of wastewater source discharge points, and query of water quality grades of major rivers and lakes in the Four-lake Basin, which makes the advanced spatial analysis of eutrophication assessment and wastewater contaminant diffusion possible. The design and implementation of the system are hence of practical significance to the water environment monitoring and management in the Fourlake Basin.

\section{Acknowledgement}

This work was funded by the (1) the Natural Science Foundation of Hubei Province, China (Grant No. 2017CFB317); (2) the Key Project of Natural Science Research in Universities of Anhui Province (Grant No. KJ2019A0763); (3) the Science and Technology Major projects of Anhui Province (18030801106, 16030801118); (4) the Key Research and Development Plan of Anhui Province, Granted (201904a07020070)

\section{References}

1. A. Watkinson, E. Murby, D. Kolpin, et al., The occurrence of antibiotics in an urban watershed: From wastewater to drinking water, Sci. Total Environ. 407(8) : 2711-2723 (2009)

2. M. Zhang, S. Wang, B. Fu, et al., Ecological effects and potential risks of the water diversion project in the Heihe River Basin, Sci. Total Environ. 619620:794-803 (2017)

3. Y. Jia, H. Zhao, C. Niu, et al., A WebGIS-based system for rainfall-runoff prediction and real-time water resources assessment for Beijing, Comput. Geosci-UK 35(7) : 1517-1528 (2009)

4. Werts, D. Joshua, et al., An integrated WebGIS framework for volunteered geographic information and social media in soil and water conservation, Environ. Manage. 49(4) : 816-832 (2012)

5. P. Wang, Z. Zou, Y. Weng, et al., Research and implementation of flood prevention and dispatching system based on WebGIS for the Yangtze River Valley, Geomatics Spatial Information Technology 32(5): 118-120 (2009)

6. P. Salvati, V. Balducci, C. Bianchi, et al., A WebGIS for the dissemination of information on historical landslides and floods in Umbria, Italy, Geoinformatica 13(3):305-322 (2009)

7. Béjar, Rubén, F. Lopez-Pellicer, J. Nogueras-Iso, et al., A protocol for machine-readable cache policies in OGC web services: Application to the EuroGeoSource information system, Environ. Modell. Softw. 60:346-356 (2014)

8. M. Yong, M. Zhang, S. Wang, et al., A Flex and ArcGIS Server based system for farmland environmental quality assessment and prediction in an agricultural producing area, Comput. Electron. Agr. 112:193-199 (2015)

9. Y. Xue, J. Liu, Real-Time water resources analysis and assessment for supporting Beijing water sustainability//Sustainability in Food and Water (Springer Netherlands, 2010)

10. M. Wang, X. Liu, J. Zhang, Evaluate method and classification standard on lake eutrophication, Environ. Monitor. China 18(5): 47-49 (2002) 